\title{
Role of the Vertical Structure of a Simulated Tropical Cyclone in Its Motion: A Case Study of Typhoon Fengshen (2008)
}

\author{
Hiroyuki Yamada ${ }^{1}$, Tomoe Nasuno ${ }^{2}$, Wataru Yanase ${ }^{3}$, and Masaki Satoh ${ }^{2,3}$ \\ ${ }^{1}$ Faculty of Science, University of the Ryukyus, Nishihara, Okinawa, Japan \\ ${ }^{2}$ Japan Agency for Marine-Earth Science and Technology, Yokohama, Kanagawa, Japan \\ ${ }^{3}$ Atmosphere and Ocean Research Institute, The University of Tokyo, Kashiwa, Chiba, Japan
}

\begin{abstract}
Typhoon Fengshen (2008) was marked by a persistent track toward the northwest, which was poorly predicted by an operational hydrostatic model, which indicated a significant northward bias. Using a global nonhydrostatic model with finer grid spacing, we have simulated a reliable track of this typhoon. The purpose of this study is to clarify the causes of the northward bias by comparing the output of the two models. This typhoon was marked by the asymmetry of rainfall concentrating in the downshear side. While both models could reproduce the asymmetric structure, a significant difference between them was found in the vertical structure. In the hydrostatic model, the vortex tilted to the downshear side with a displacement from lower to upper levels exceeding $100 \mathrm{~km}$. This tilt was related to weak updrafts of, at most, $0.5 \mathrm{~m} \mathrm{~s}^{-1}$. Diagnosis using vorticity budget demonstrated that the tilt of the vortex resulted from a lack of vertical coupling that was too weak to withstand differential advection between the lower and upper levels. These results suggest the importance of reproducing inner-core updrafts for better track prediction of a typhoon in an environment with strong vertical shear.
\end{abstract}

(Citation: Yamada, H., T. Nasuno, W. Yanase, and M. Satoh, 2016: Role of the vertical structure of a simulated tropical cyclone in its motion: A case study of Typhoon Fengshen (2008). SOLA, 12, 203-208, doi:10.2151/sola.2016-041.)

\section{Introduction}

The forecasting of tropical cyclone (TC) movement is important for disaster prevention and mitigation. The accuracy of track prediction has improved greatly over the last few decades due to improvements in numerical prediction models (Heming and Goerss 2010). However, significant errors in track prediction, exceeding $1,000 \mathrm{~km}$ over 3 days, still exist and prevent further reduction of the annual-mean error (Yamaguchi et al. 2012). Typhoon Fengshen (2008) was a case of poor track forecasting, with a position error of up to $1,500 \mathrm{~km}$ over 5 days against the observed track. In Fig. 1a, each blue segment shows the track predicted from 1200 UTC of each day by the global spectral model (GSM) of the Japan Meteorological Agency (JMA). All of the predicted tracks deviate northward from the observed track (green). Elsberry et al. (2010) pointed out that this northward bias for Fengshen was common to all of the deterministic model guidance available to the Joint Typhoon Warning Center (JTWC), and this led to some of the largest forecast errors of the 2008 season. Cooper and Falvey (2008) stated that immediate evaluation by the modeling community is necessary to determine the root causes of the unreliability of the dynamic models in this case. Recently, the Eighth International Workshop on Tropical Cyclones (IWTC-VIII) recommended that the TC research community explore causes of large forecast track errors (WMO 2014). Moreover, this typhoon caused severe floods and landslides during passage across the Philippines, with damages of more than 252 million US dollars

Corresponding author: Hiroyuki Yamada, Faculty of Science, University of the Ryukyus, 1 Senbaru, Nishihara, Okinawa 903-0213, Japan. E-mail: yamada@sci.u-ryukyu.ac.jp. (C2016, the Meteorological Society of Japan.
(Yumul et al. 2012). However, the cause of the track error has not yet been clarified.

TC motion is mostly due to steering (i.e., horizontal advection of relative vorticity), the beta effect (meridional advection of the Coriolis parameter), and their nonlinear interaction, as summarized by Chan (2010). In the Fengshen case, the serious errors in operational prediction imply considerable effects of other processes that cannot be resolved in the operational models on TC movement. One of the processes that needs to be examined is the influence of the asymmetric structure on its motion. Chen et al. (2014) described that Fengshen was accompanied by persistent mesoscale convective systems to the west of the vortex center. Previous studies showed a relationship between TC asymmetry and the deep-tropospheric $(850-200 \mathrm{hPa})$, mean vertical shear in its environment (Ueno 2007; Corbosiero and Molinari 2002). The contribution of asymmetric convection to TC motion was demonstrated in some of previous studies (Wu and Wang 2001; Chan et al. 2002). It is necessary to understand the importance of asymmetric convection and vertical wind shear relative to the steering and beta effects on the motion of Fengshen.

In the present study, Fengshen was numerically simulated using the Nonhydrostatic Icosahedral Atmospheric Model (NICAM, Satoh et al. 2008, 2014) with 3.5-km horizontal grid spacing and without any cumulus parameterization scheme (Hashino et al. 2013). This model has been used for research on TC genesis and intensity (e.g., Yanase et al. 2010; Satoh et al. 2012; Kodama et al. 2015). The resolution of operational global models is rapidly increasing ( $\sim 20 \mathrm{~km}$ or finer), and soon it will be finer than $10 \mathrm{~km}$, where a nonhydrostatic model is more adequate to resolve convective-scale processes. It is necessary to understand and improve the performance of a global nonhydrostatic model with a cloud-permitting horizontal resolution using individual TC cases. The purposes of this study are to clarify the leading processes of the movement of Fengshen and to identify the major cause of the northward bias of the operational prediction models by comparing the outputs of NICAM and JMA-GSM.

\section{Numerical models and data}

The experiment design of the NICAM simulation was described in Hashino et al. (2013). The domain of NICAM covers the whole globe with $3.5-\mathrm{km}$ horizontal spacing and consists of 40 vertical layers up to $38 \mathrm{~km}$ mean sea level (MSL). The moist processes were explicitly calculated using a cloud microphysical scheme with six categories of water species (NSW6, Tomita 2008). The simulation was initialized at 0000 UTC 15 June 2008, 4 days prior to Fengshen's designation as a tropical storm, using the European Centre for Medium-Range Weather Forecasts (ECMWF)-Year of Tropical Convection (YOTC) operational data with $0.5^{\circ} \times 0.5^{\circ}$ horizontal resolution. The simulation was performed for 10 days as a free run. At the bottom boundary, the sea surface temperature (SST) was predicted using a slab ocean model and was nudged to weekly Optimum Interpolation SST data (Reynolds and Smith 1994) at a relaxation time of 5 days. The simulation was performed using the Earth Simulator supercomputer of the Japan Agency for Marine-Earth Science and Technology.

GSM predictions were operationally performed by JMA every 
12 hours (Nakagawa 2009). The model has horizontal grid spacing of $20 \mathrm{~km}$ and consists of 60 pressure layers of up to $0.1 \mathrm{hPa}$. The output was provided by JMA after resampling with grid spacing of $50 \mathrm{~km}$.

The observational data used in this study are the best track record of the typhoon issued by the regional specialized meteorological center (RSMC) in Tokyo, infrared imagery observed by the JMA's MTSAT-1R satellite, and the surface rain rate over oceans estimated using the Special Sensor Microwave Imager (SSM/I) on board the Defense Meteorological Satellite Program (DMSP) satellites, provided by Remote Sensing Systems.

\section{Horizontal and vertical structure of Fengshen in the two models}

To describe the reproducibility of Fengshen by NICAM, the simulated track is indicated by a red line in Fig. 1a. This track is persistently directed toward the northwest, similar to the observed track (green line). The position error at 120 hours from initialization (i.e., 0000 UTC 20 June) was $277 \mathrm{~km}$, approximately a half of the annual-mean error of JMA-GSM $(\sim 500 \mathrm{~km}$, Yamaguchi et al. 2012). Horizontal distributions of the satellite-derived cloudtop temperature and the surface rain rate at 2100 UTC 18 June are shown in Figs. 1b and 1c, respectively. Within $200 \mathrm{~km}$ of the center, deep cloud areas $\left(<65^{\circ} \mathrm{C}\right)$ with intense rainfall $(>8.0 \mathrm{~mm}$ $\mathrm{hr}^{-1}$ ) were concentrated in the southwestern quadrant. Beyond the $200 \mathrm{~km}$ range, a spiral rainband developed on the western to northwestern side. This significant asymmetry was persistently observed afterwards, as reported by Chen et al. (2014). The NICAM simulation at 0000 UTC 20 June (Fig. 2a) succeeded in reproducing the asymmetric structure, with intense rainfall $(>$ $8.0 \mathrm{~mm} \mathrm{~h}^{-1}$ ) on the southern side, within the $200-\mathrm{km}$ range, and the outer rainband on the west to northern side. The horizontal divergence at $1.6 \mathrm{~km}$ MSL (Fig. 2b) was calculated from winds smoothed using a Gaussian filter of 200-km width for comparison with the results of JMA-GSM. Negative values (i.e., convergence) were mainly distributed on the western side of the center, implying the concentration of lower-level convergence with updrafts aloft to the southwest of the TC center.

A relationship between the asymmetric structure and environmental wind was examined using the vertical shear calculated between $1^{\circ}$ and $8^{\circ}$ horizontal radii and between the 1.6-km (near $850 \mathrm{hPa}$ ) and 12.0-km (near $200 \mathrm{hPa}$ ) vertical layers. The vertical shear (shown by a red arrow in Figs. 2a and 2b) was $12.6 \mathrm{~m} \mathrm{~s}^{-1}$ toward the southwest, approximately $90^{\circ}$ left of the typhoon track (blue arrow). The rainfall peak in the inner $(<100 \mathrm{~km})$ region existed on the downshear-left side, similar to the results of Ueno (2007). The rainfall peak in the outer $(100-300 \mathrm{~km})$ region existed on the downshear-right side, similar to Corbosiero and Molinari (2002). These results suggest the contribution of the environmental vertical shear to the asymmetric structure.

Next, the reproducibility by JMA-GSM is examined using the prediction initialized at 1200 UTC 18 June (highlighted in yellow in Fig. 1a), which is used for detailed analyses in this study. The predicted track was initially directed to the northwest and turned to the north-northwest around 0000 UTC 20 June (i.e., 36 hours from initialization). The rainfall distribution at this hour (Fig. 2c) is marked by concentration of intense rain $\left(>8.0 \mathrm{~mm} \mathrm{~h}^{-1}\right)$ on the southwestern side. The distribution of divergence at $850 \mathrm{hPa}$ (Fig. $2 \mathrm{~d}$ ), calculated from smoothed winds, shows the convergence to the west of the TC center. The shear vector, calculated between 850 and $200 \mathrm{hPa}$, was directed southwestward. It is noteworthy that the asymmetric structure was significant even in JMA-GSM, although the magnitude of convergence $\left(\sim 0.4 \times 10^{-4} \mathrm{~s}^{-1}\right)$ was much smaller than that in NICAM $\left(\sim 2 \times 10^{-4} \mathrm{~s}^{-1}\right.$, Fig. 2b).

To examine the vertical structure, cross sections of wind vectors and relative vorticity along a line parallel to the vertical shear vector are shown in Fig. 3. The vortex in NICAM was nearly upright, with displacement of the upper-level $(>10 \mathrm{~km}$ MSL) circulation center of, at most, $10 \mathrm{~km}$ on the downshear side. The magnitude of updrafts in the eyewall was up to $7 \mathrm{~m} \mathrm{~s}^{-1}$. In contrast, the vortex in JMA-GSM significantly tilted to the downshear side with height. The circulation center was displaced more than $100 \mathrm{~km}$ above $10 \mathrm{~km}$ MSL. Updrafts were $0.5 \mathrm{~m} \mathrm{~s}^{-1}$ at most, which is too weak for a TC updraft. The track of the circulation center at $300 \mathrm{hPa}$ in JMA-GSM is shown in Fig. 2d. This position was persistently displaced about $100 \mathrm{~km}$ to the downshear side of the surface center during the northward movement. Although the downshear tilt is reported in previous studies, horizontal displacement of the vortex is usually limited to within $15 \mathrm{~km}$ between the lower and upper troposphere (e.g., Reasor et al. 2000; Rogers et al. 2003). Since upward motion (with diabatic heating) is important for vertical coupling of TC circulation (e.g., Wu and Wang 2001), it is suggested that the vertical tilt resulted from the underestimated updrafts.

The steering level of the TC center is examined using the hodograph of environmental winds (Fig. 4). The TC motion in

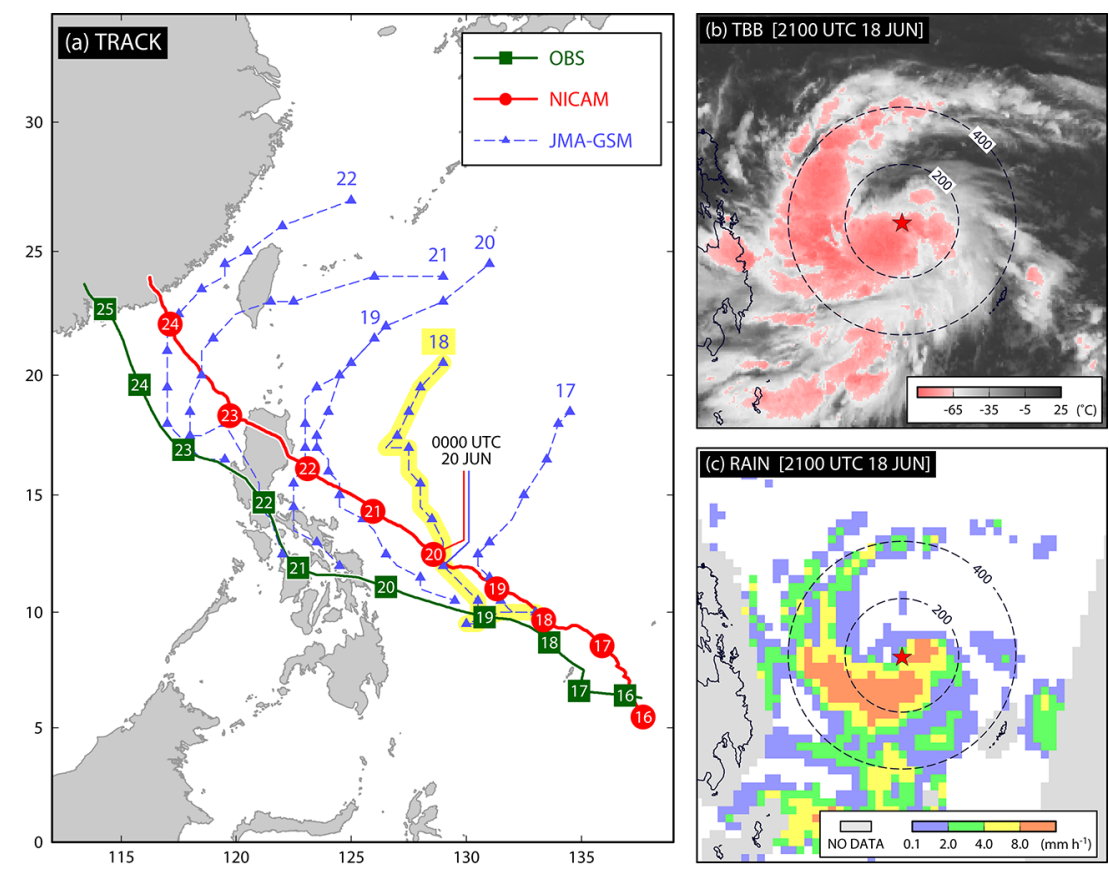

Fig. 1. (a) Observed and simulated tracks of Typhoon Fengshen (2008). Each green square (red circle) with a number indicates the typhoon center observed (simulated by NICAM) at 0000UTC of each day. As for JMAGSM, tracks simulated from 1200 UTC of each day are drawn in blue, with triangles for the 12-hourly center position. Number indicates the date of initialization. (b) Horizontal distribution of the infrared cloud-top temperature observed by MTSAT-1R at 2100 UTC 18 June. (c) Distribution of the microwave-derived rain rate by DMSP SSM/I F17 satellite at 2100 UTC 18 June. 

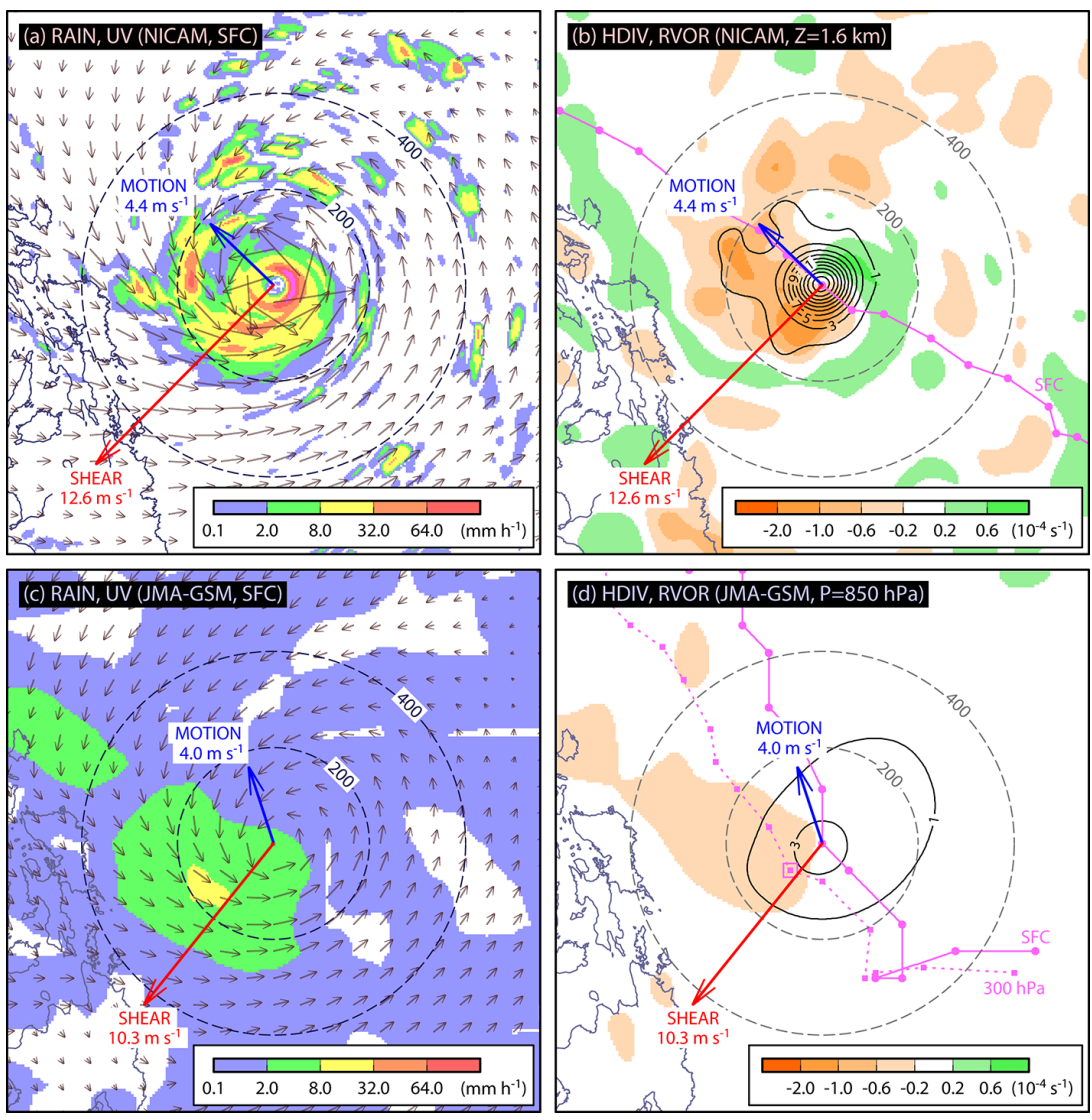

Fig. 2. Horizontal distributions at 0000 UTC 20 June simulated by NICAM (top) and JMA-GSM (bottom). The left panels show the rain rate (shade) and surface wind vectors. The right panels show the horizontal divergence (shade) and the relative vorticity (contour) at $1.6 \mathrm{~km} \mathrm{MSL}$ or $850 \mathrm{hPa}$. Broken circles indicate $200-$ and $400-\mathrm{km}$ ranges from the surface vortex center. A blue (red) arrow indicates the TC motion and vertical shear. The 6-hourly positions of the center at surface $(300 \mathrm{hPa})$ are shown by circles with solid lines (rectangles with dotted lines).
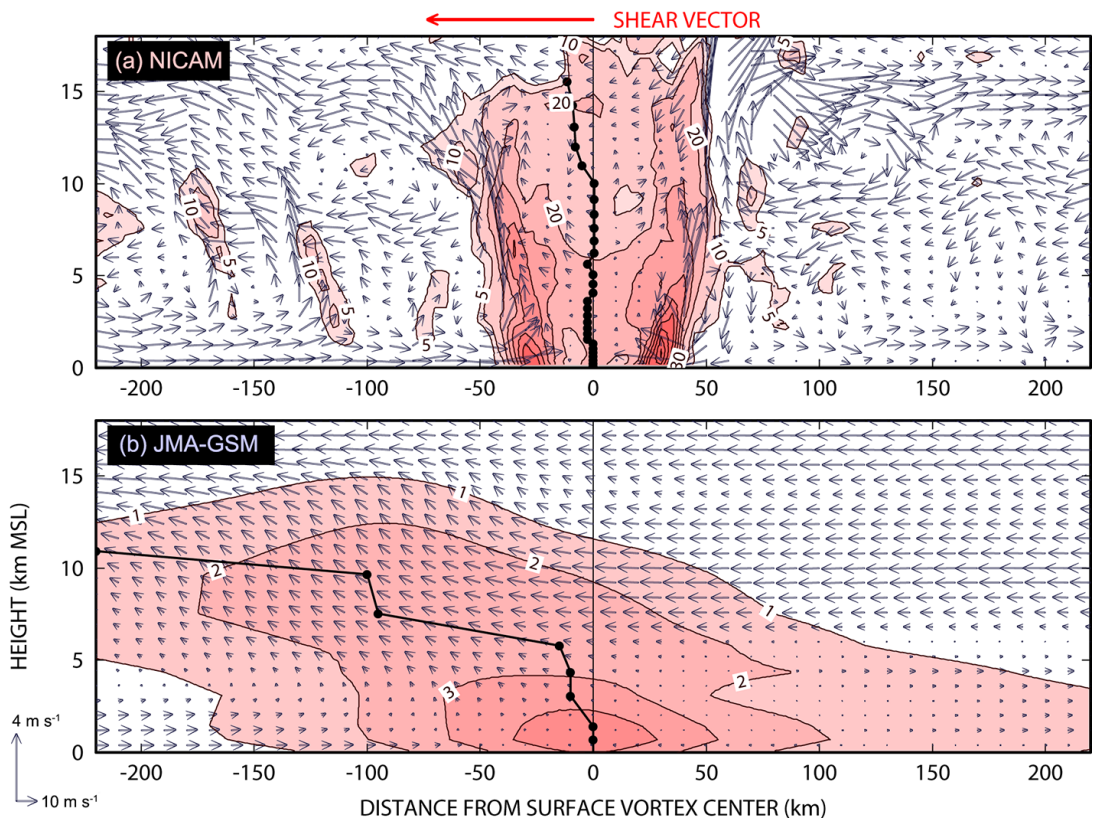

Fig. 3. Vertical cross sections of wind vectors and relative vorticity (unit of $10^{-4} \mathrm{~s}^{-1}$ ) at 0000 UTC 20 June in the direction parallel to the vertical shear vector, simulated by (a) NICAM and (b) JMA-GSM. Location of the circulation center at each layer is shown by a solid circle.

NICAM corresponds to the environmental winds near $6 \mathrm{~km}$, while that in JMA-GSM corresponds to winds near $600 \mathrm{hPa}$ (i.e., $4 \mathrm{~km}$ ). This suggests that the upright vortex in NICAM was steered by mid-tropospheric winds, while the lower part of the tilted vortex in JMA-GSM was steered by lower-tropospheric winds.

\section{Vorticity budget analysis}

To quantitatively diagnose the role of the asymmetric and vertical structure in TC motion, a vorticity budget analysis was employed. Although a potential vorticity tendency diagnosis (Wu and Wang 2001) is preferable to this analysis, we employed the vorticity budget due to lack of diabatic heating in the output of JMA-GSM. Based on the equation of absolute vertical vorticity $(\zeta+f)$ in the Cartesian coordinate (e.g., Tory et al. 2006; Kieu and Zhang 2009), the tendency of vorticity can be represented as the following equation if friction and other minor terms can be ignored. 


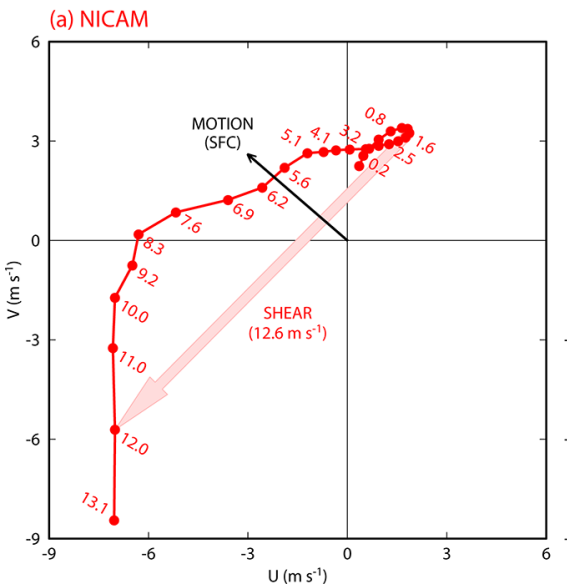

(b) JMA-GSM

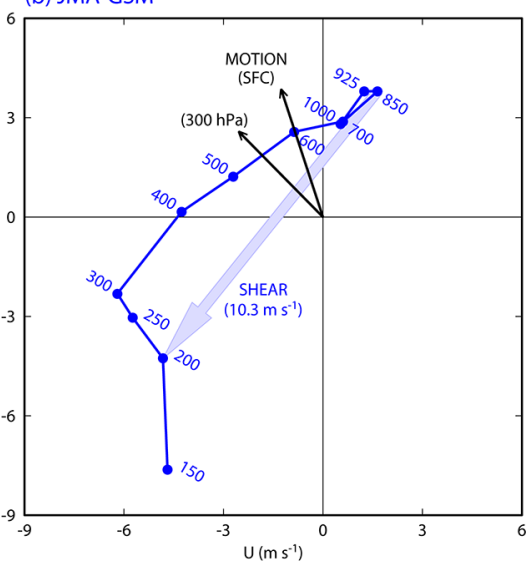

Fig. 4. Hodograph of environmental winds below 150 $\mathrm{hPa}$ at 0000 UTC 20 June, averaged between $1^{\circ}$ and $8^{\circ}$ radii using the results of (a) NICAM and (b) JMAGSM. Thin arrows show the motion of the circulation center, and a thick arrow shows the vertical shear between 1.6 and $12.0 \mathrm{~km}$ (or 850 and $200 \mathrm{hPa}$ ).

$$
\begin{aligned}
\frac{\partial(\zeta+f)}{\partial t}= & -\underbrace{\left(u \frac{\partial \zeta}{\partial x}+v \frac{\partial \zeta}{\partial y}+w \frac{\partial \zeta}{\partial z}\right)}_{\text {Advection }}-\underbrace{v \frac{\partial f}{\partial y}}_{\text {Coriolis }} \\
& -\underbrace{(\zeta+f)\left(\frac{\partial u}{\partial x}+\frac{\partial v}{\partial y}\right)}_{\text {Stretching }}-\underbrace{\left(\frac{\partial w}{\partial x} \frac{\partial v}{\partial z}-\frac{\partial w}{\partial y} \frac{\partial u}{\partial z}\right)}_{\text {Tilting }}
\end{aligned}
$$

Terms on the right-hand side represent the horizontal and vertical advection of the relative vorticity $(\zeta)$, advection of Coriolis parameter $(f)$, stretching, and tilting. The horizontal advection (hereafter, HADV) represents the steering of a vortex by environmental horizontal winds, while the vertical advection (VADV) means vertical extension of the vortex due to vertical motion. The effect of horizontal convergence is included in the stretching term (VSTR). The total tendency (TEND) in the left-hand side was estimated by summing all the terms on the right-hand side. If a positive-vorticity area moves in a direction, a positive maximum of TEND should exist in that direction. In the calculation of each term, wind components smoothed using a Gaussian filter of 250-km width were used.

The horizontal distributions of each term at 1.6 and $9.2 \mathrm{~km}$ MSL at 0000 UTC 20 June, simulated by NICAM, are shown in Fig. 5. The Coriolis and tilting terms were more than an order of magnitude smaller than the tendency (not shown), meaning negligible impact of the beta effect on TC motion. In both levels, a positive peak of TEND exists in the same direction as the TC motion toward the northwest. In contrast, positive HADV peaks deviate from the motion vector in both levels, according to the northward (westward) steering effect at 1.6 (9.2) km (see Fig. 4). This differential advection was compensated by a positive VSTR in the lower level and by a combination of VADV and VSTR in the upper level. Since both VADV and VSTR relate to vertical motion, it is suggested that a vertical coupling of the vortex due to inner-core updrafts was important for sustaining the vertical coherent structure against strong vertical shear.

The distributions at the same hour simulated by JMA-GSM are shown in Fig. 6. Note that the contour interval is $3 \times 10^{-9} \mathrm{~s}^{-2}$, an order of magnitude smaller than that in Fig. 5. The location

$\operatorname{NICAM}(Z=1.6 \mathrm{~km})$
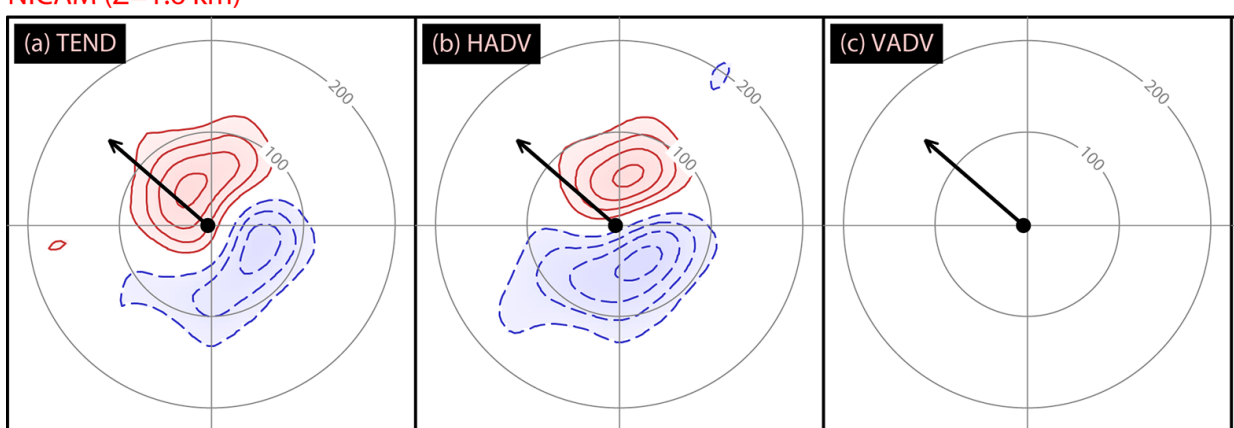

[CONTOUR INTERVAL: $3 \times 10^{-8} \mathrm{~s}^{-2}$ ]

$\operatorname{NICAM}(Z=9.2 \mathrm{~km})$
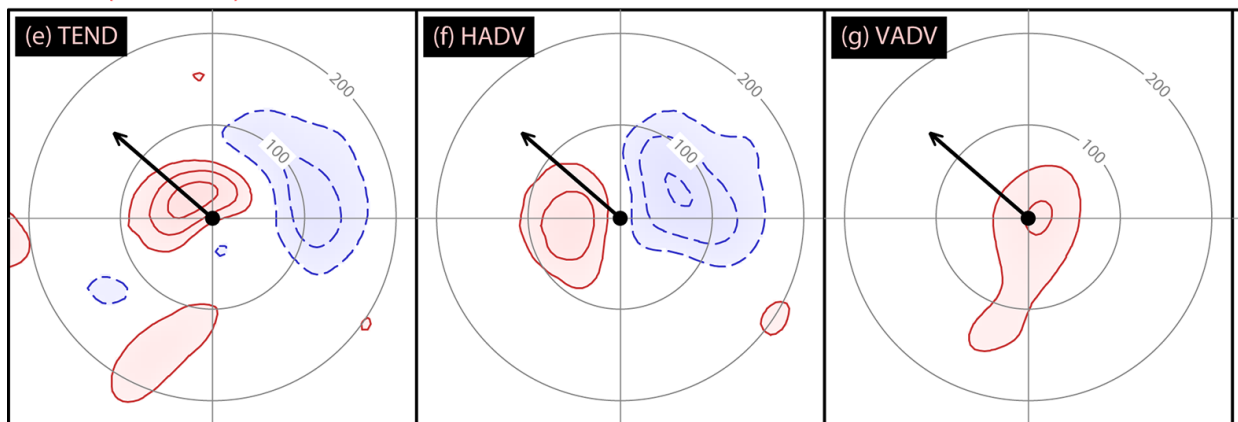

[CONTOUR INTERVAL: $3 \times 10^{-8} \mathrm{~s}^{-2}$ ]

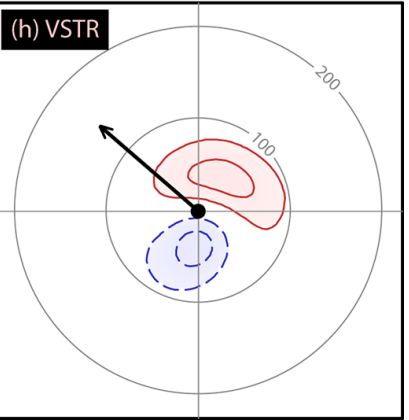

Fig. 5. Horizontal distributions of the total tendency (TEND), horizontal advection (HADV), vertical advection (VADV), and stretching (VSTR) calculated using the output of NICAM at 1.6 and $9.2 \mathrm{~km} \mathrm{MSL}$ at 0000 UTC 20 June. Positive (negative) contours are drawn in red (blue) with interval $3 \times 10^{-8} \mathrm{~s}^{-2}$. The zero contours are omitted. A solid circle with an arrow indicates the position and moving direction of the circulation center at each level. 
JMA-GSM (P=850 hPa)
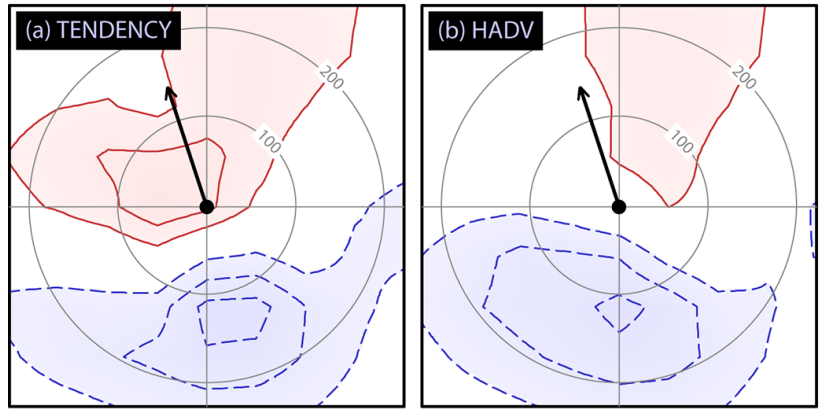

JMA-GSM ( $\mathrm{P}=300 \mathrm{hPa})$
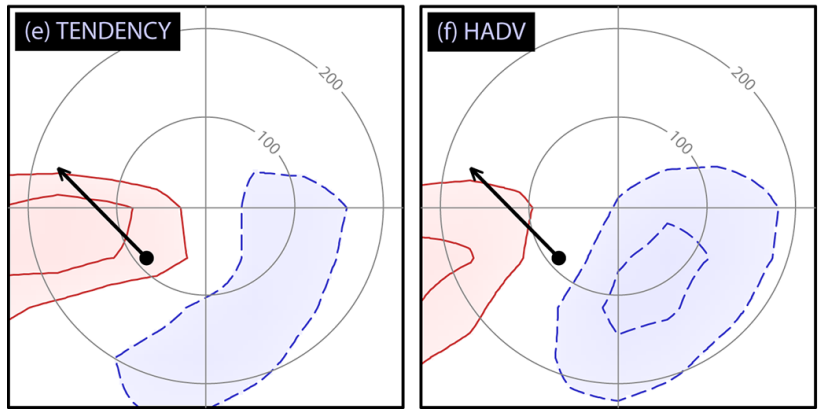
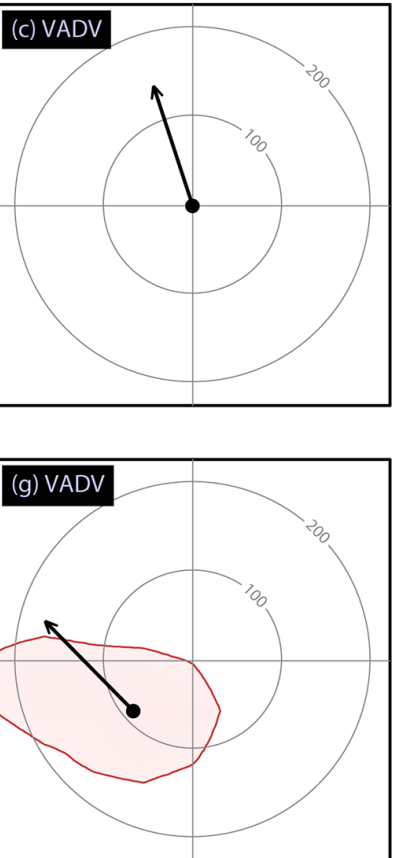

[CONTOUR INTERVAL: $3 \times 10^{-9} \mathrm{~s}^{-2}$ ]

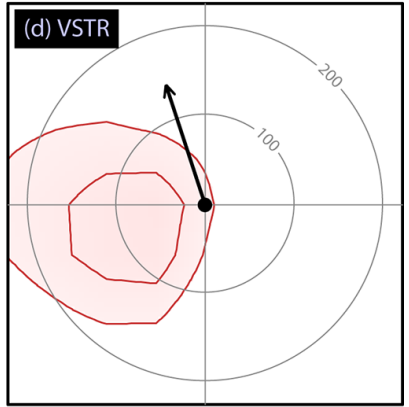

[CONTOUR INTERVAL: $3 \times 10^{-9} \mathrm{~s}^{-2}$ ]

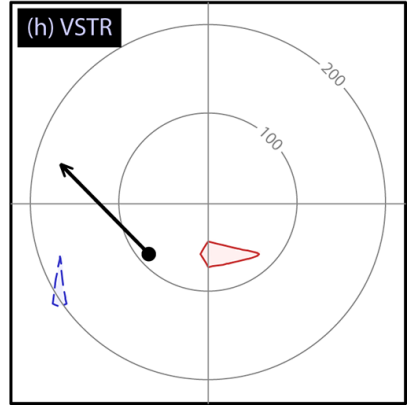

Fig. 6. Same as Fig. 5, but using the output of JMA-GSM at 850 and $300 \mathrm{hPa}$. Contour interval is $3 \times 10^{-9} \mathrm{~s}^{-2}$.

of positive TEND peaks in the upper level deviates from that in lower level, according to the separation of the vortex center. In both levels, the location of a positive HADV peak roughly corresponded to that of the TEND peak. A positive peak of VSTR (VADV) in the lower (upper) levels existed near the upper-level vortex center. Although VSTR and VADV have a magnitude comparable with HADV, these values are less than one-tenth of those in the NICAM simulation (Fig. 5). It is suggested that the weak vertical coupling due to weak updrafts allowed the predominance of different advection, causing the tilted structure and the northward bias of the lower-level vortex center.

\section{Simulated vertical structure throughout the life- cycle}

Figure 7 highlights the difference between the vertical structure of NICAM and JMA-GSM throughout the lifecycle by showing the time series of the horizontal distance between the lower and upper circulation centers. In NICAM, the distance was within $15 \mathrm{~km}$ from 19 to 21 June. This implies that the vortex maintained an upright structure against the vertically sheared environment until making landfall in the Philippines (Fig. 1). In contrast, individual JMA-GSM simulations show continuous increase in the distance. The simulation from 12 UTC on 18 June (highlighted in yellow) shows that the displacement was persistently greater than $100 \mathrm{~km}$ after 0000 UTC 20 June. This displacement corresponds to a tilt toward the downshear side (Fig. 2d). The vertical velocity at $500 \mathrm{hPa}$ (not shown) was smaller than $1 \mathrm{~m} \mathrm{~s}^{-1}$ in all of the vortices in JMA-GSM. These results suggest that a vertically tilted structure was common in all simulated typhoons with a northward bias.

\section{Summary and discussion}

This study examined the role of the vertical structure of Typhoon Fengshen (2008) in its motion using the nonhydrostatic model NICAM to clarify the cause of the northward bias of motion predicted by an operational hydrostatic model (JMAGSM). The observed typhoon was characterized by a persistence

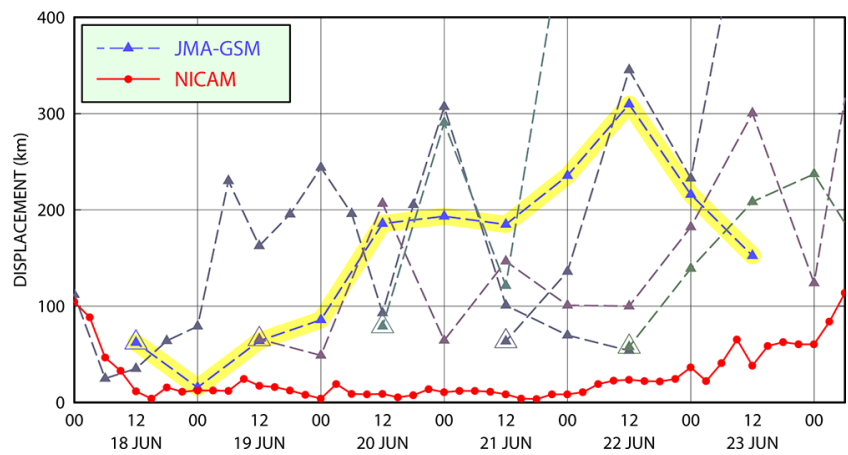

Fig. 7. Time series of the horizontal displacement of the circulation center between 1.6 and $9.2 \mathrm{~km}$ MSL simulated by NICAM (solid circles) and between 850 and $300 \mathrm{hPa}$ simulated by JMA-GSM (solid triangles). In JMA-GSM simulations, each open triangle indicates the initial hour.

of asymmetry with the concentration of intense rainfall in the southwestern quadrant. Both models succeeded in reproducing the asymmetric structure with a concentration of rain and updrafts on the downshear side. However, the hydrostatic model failed to reproduce the upright vertical structure, with the horizontal displacement of the vortex center exceeding $100 \mathrm{~km}$ between the lower and upper troposphere. A vorticity budget analysis demonstrated that the tilt of the vortex resulted from a lack of vertical coupling that was too weak to withstand the differential vertical shear effect. This tilt allowed the lower part of the vortex to move with a southerly flow in the lower troposphere, causing the northward track error.

These results suggest that the contributions of vertical wind shear and asymmetric diabatic heating must be taken into account for the motion of Fengshen. Chan et al. (2002) showed that asymmetric heating is not negligible for the motion of asymmetric TCs while it is less important for that of axisymmetric TCs. Therefore, the accurate reproducibility of horizontally asymmetric, vertically upright structure from the initial hour in a case under a strong vertical shear $\left(>10 \mathrm{~m} \mathrm{~s}^{-1}\right)$ is necessary to further improve operational track prediction. Since the global non-hydrostatic model requires 
huge computational resources, the use of a regional model with explicit cloud microphysical processes, with a horizontal grid spacing smaller than several kilometers, may be an efficient approach.

Although this study has provided a single case, there are other examples of northward bias, including Typhoon Morakot (2009) and Typhoon Parma (2009), as reported in Yamaguchi et al. (2012). $\mathrm{Wu}$ and $\mathrm{Wu}$ (2011) reported the highly asymmetric structure of Morakot, which is similar to that of Fengshen. Analyses of the vertical structure of these typhoons will contribute to a better understanding of the role of vertical structure in TC motion.

\section{Acknowledgments}

The authors express their gratitude to two reviewers for providing valuable and constructive comments that were useful for improving the original manuscript. The output of JMA-GSM was provided by the Research Institute for Sustainable Humanosphere, Kyoto University (http://database.rish.kyoto-u.ac.jp/arch/ jmadata/). The MTSAT-1R satellite image was provided by Weather Home, Kochi University (http://weather.is.kochi-u.ac.jp/). The SSM/I dataset was produced by Remote Sensing Systems (http://www.remss.com). Figures were drawn using Generic Mapping Tools (https://www.soest.hawaii.edu/gmt/). The authors HY and TN are supported by JSPS KAKENHI Grant Number $16 \mathrm{H} 04053$ and 26400475 , respectively.

Edited by: H. Kamahori

\section{References}

Chan, J. C. L., F. M. F. Ko, and Y. M. Lei, 2002: Relationship between potential vorticity tendency and tropical cyclone motion. J. Atmos. Sci., 59, 1317-1336.

Chan, J. C. L., 2010: Movement of tropical cyclones. Global Perspectives on Tropical Cyclones, J. C. L. Chan and J. D. Kepert, Eds., World Sci., Singapore, 133-148.

Chen, B.-F., R. L. Elsberry, and C.-S. Lee, 2014: Origin and maintenance of the long-lasting, outer mesoscale convective system in Typhoon Fengshen (2008). Mon. Wea. Rev., 142, 2838-2859, doi:10.1175/MWR-D-14-00036.1.

Cooper, G. A., and R. J. Falvey, 2008: Annual tropical cyclone report. US Naval Maritime Forecast Center/Joint Typhoon Warning Center Pearl Harbor, Hawaii.

Corbosiero, K. L., and J. Molinari, 2002: The effects of vertical wind shear on the distribution of convection in tropical cyclones. Mon. Wea. Rev., 130, 2110-2123, doi:10.1175/15200493(2002)130<2110:TEOVWS >2.0.CO;2.

Elsberry, R. L., M. S. Jordan, and F. Vitart, 2010: Predictability and tropical cyclone events on intraseasonal timescales with the ECMWF monthly forecast model. Asia-Pacific J. Atmos. Sci., 46, 135-153, doi:10.1007/s13143-010-0013-4.

Hashino, T., M. Satoh, Y. Hagihara, T. Kubota, T. Matsui, T. Nasuno, and H. Okamoto, 2013: Evaluating cloud microphysics from NICAM against CloudSat and CALIPSO. J. Geophys. Res. Atmos., 118, 7273-7292, doi:10.1002/jgrd.50564.

Heming, J., and J. Goerss, 2010: Track and structure forecasts of tropical cyclones. Global Perspectives on Tropical Cyclones, J. C. L. Chan and J. D. Kepert, Eds., World Sci., Singapore, 133-148.

Kieu, C. Q., and D.-L. Zhang, 2009: Genesis of Tropical Storm Eugene (2005) from merging vortices associated with ITCZ breakdowns. Part II: Roles of vortex merger and ambient potential vorticity. J. Atmos. Sci., 66, 1980-1996.

Kodama, C., Y. Yamada, A. T. Noda, K. Kikuchi, Y. Kajikawa, T. Nasuno, T. Tomita, T. Yamaura, T. G. Takahashi, M. Hara, Y. Kawatani, M. Satoh, and M. Sugi, 2015: A 20-year climatology of a NICAM AMIP-type simulation. J. Meteor. Soc. Japan, 93, doi:10.2151/jmsj.2015-024.
Nakagawa, M., 2009: Outline of the high resolution global model at the Japan Meteorological Agency. RSMC Tokyo-Typhoon Center Technical Review, 11, JMA, Japan, 1-13 (Available online at http://www.jma.go.jp/jma/jma-eng/jma-center/rsmchp-pub-eg/techrev.htm, accessed on 26 June 2016).

Reasor, P. D., M. T. Montgomery, F. D. Marks Jr., and J. F. Gamache, 2000: Low-wavenumber structure and evolution of the hurricane inner core observed by airborne dual-Doppler radar. Mon. Wea. Rev., 128, 1653-1680.

Reynolds, R. W., and T. M. Smith, 1994: Improved global sea-surface temperature analyses using optimum interpolation. J. Climate, 7, 929-948, doi:10.1175/1520-0442(1994)007<0929:IGSSTA $>2.0 . \mathrm{CO} ; 2$.

Rogers, R., S. Chen, J. Tenerelli, and H. Willoughby, 2003: A numerical study of the impact of vertical shear on the distribution of rainfall in Hurricane Bonnie (1998). Mon. Wea. Rev., 131, 1577-1599.

Satoh, M., T. Matsuno, H. Tomita, H. Miura, T. Nasuno, and S. Iga, 2008: Nonhydrostatic Icosahedral Atmospheric Model (NICAM) for global cloud resolving simulations. J. Comp. Phys., 227, 3486-3514, doi:10.1016/j.jcp.2007.02.006.

Satoh, M., K. Oouchi, T. Nasuno, H. Taniguchi, Y. Yamada, H. Tomita, C. Kodama, J. Kinter, D. Achuthavarier, J. Manganello, B. Cash, T. Jung, T. Palmer, and N. Wedi, 2012: The intra-seasonal oscillation and its control of tropical cyclones simulated by high-resolution global atmospheric models. Climate Dyn., 39, 2185-2206, doi:10.1007/s00382-011-1235-6.

Satoh, M., H. Tomita, H. Yashiro, H. Miura, C. Kodama, T. Seiki, A. T. Noda, Y. Yamada, D. Goto, M. Sawada, T. Miyoshi, Y. Niwa, M. Hara, T. Ohno, S. Iga, T. Arakawa, T. Inoue, and H. Kubokawa, 2014: The non-hydrostatic icosahedral atmospheric model: Description and development. Prog. Earth Planet. Sci., 1, 18. doi:10.1186/s40645-014-0018-1.

Tomita, H., 2008: New micorphysical schemes with five and six categories by diagnostic generation of cloud ice. J. Meteor. Soc. Japan, 86A, 121-142. doi:10.2151/jmsj.86A.121.

Ueno, M., 2007: Observational analysis and numerical evaluation of the effects of vertical wind shear on the rainfall asymmetry in the typhoon inner-core region. J. Meteor. Soc. Japan, 85, 115136, doi:10.2151/jmsj.85.115.

Tory, K. J., M. T. Montgomery, and N. E. Davidson, 2006: Prediction and diagnosis of tropical cyclone formation in an NWP system. Part I: The critical role of vortex enhancement in deep convection. J. Atmos. Sci., 63, 3077-3090.

WMO, 2014: Recommendations addressed to both operational centres and research community. (Available online at http:// www.wmo.int/pages/prog/arep/wwrp/tmr/documents/Listof Recommendations.pdf, accessed on 12 June 2016).

Wu, L., and B. Wang, 2001: Effects of convective heating on movement and vertical coupling of tropical cyclones: A numerical study. J. Atmos. Sci., 58, 3639-3649.

Wu, L., J. Liang, and C.-C. Wu, 2011: Monsoonal influence on Typhoon Morakot (2009). Part I: Observational analysis. $J$. Atmos. Sci., 68, 2208-2221. doi:10.1175/2011JAS3730.1.

Yamaguchi, M., T. Nakazawa, and K. Aonashi, 2012: Tropical cyclone track forecasts using JMA model with ECMWF and JMA initial conditions. Geophys. Res. Lett., 39, L09801, doi: 10.1029/2012GL051473.

Yanase, W., M. Satoh, H. Yamada, K. Yasunaga, and Q. Moteki, 2010: Continual influences of tropical waves on the genesis and rapid intensification of Typhoon Durian (2006). Geophys. Res. Lett., 37, L08809, doi:10.1029/2010GL042516.

Yumul. G. P. Jr., N. T. Servando, L. O. Suerte, M. Y. Magarzo, L. V. V. Juguan, and C. B. Dimalanta, 2012: Tropical cyclonesouthwest monsoon interaction and the 2008 floods and landslides in Panay island, central Philippines: Meteorological and geological factors. Nat. Hazards, 62, 827-840, doi:10.1007/ s11069-012-0109-5.

Manuscript received 31 March 2016, accepted 22 June 2016

SOLA: https://www.jstage.jst.go.jp/browse/sola/ 